\title{
Obliquity Pacing of the Late Pleistocene Glacial Terminations
}

\section{Citation}

Huybers, Peter J., and Carl Wunsch. 2005. Obliquity pacing of the late Pleistocene glacial terminations. Nature 434: 491-494.

\section{Published Version}

http://dx.doi.org/10.1038/nature03401

\section{Permanent link}

http://nrs.harvard.edu/urn-3:HUL.InstRepos:3382978

\section{Terms of Use}

This article was downloaded from Harvard University's DASH repository, and is made available under the terms and conditions applicable to Other Posted Material, as set forth at http:// nrs.harvard.edu/urn-3:HUL.InstRepos:dash.current.terms-of-use\#LAA

\section{Share Your Story}

The Harvard community has made this article openly available.

Please share how this access benefits you. Submit a story.

Accessibility 
running and when the locking loop is closed. The closed-loop frequency fluctuations exhibit a root-mean-square (r.m.s.) frequency deviation of $\sim 310 \mathrm{kHz}$ over a 1-min integration time. The residual noise exhibits a white noise spectrum with an Allan variance of $3 \times 10^{-11} / \tau^{1 / 2}$ for an averaging time of $1 \mathrm{~s} \leq \tau \leq 25.6 \mathrm{~s}$, and is limited by the electronic noise of our detection system (PD3 in Fig. 4a).

A straightforward improvement would be to use a saturable absorption line (which can have a linewidth as narrow as $1 \mathrm{MHz}$; ref. 13) as a reference. The improved signal-to-noise ratio in HC-PCF also makes overtone absorptions in the visible and near-infrared (for example, $\mathrm{P} 11$ of ${ }^{12} \mathrm{C}_{2} \mathrm{H}_{2}$ at $790.703 \mathrm{~nm}$ ) accessible to laser frequency metrology. To test for gas leakage, the pressuredependent linewidth of the P9 acetylene absorption was monitored daily over two months. The results gave an average value of $\sim 468 \mathrm{MHz}$ with a standard deviation of $30 \mathrm{MHz}$, that is, the linewidth is consistent with the Doppler limited value and was constant within experimental error, indicating no measurable leakage of gas.

In conclusion, all-fibre, ultra-compact, high performance, easyto-use and unconditionally stable gas-laser devices have been reported. The commercial availability of a wide range of all-fibre components (for example, lasers, phase modulators, power attenuators, isolators, Bragg gratings and beam-splitters) makes complex systems easy to design and construct. It is now possible to imagine miniature laser-gas devices, occupying a tiny volume and containing minute amounts of gas, with everyday applications in fields such as the colour conversion of laser light (perhaps using a built-in diode pump laser), and the measurement and stabilization of laser frequency. The unique features of HC-PCFs make these gas-laser devices extremely efficient, and their impact in many laser-related fields is likely to be deep and lasting.

\section{Methods}

\section{Splicing procedure}

Fusion-splicing was carried out using a commercial filament-based splicer (Vytran FFS-2000-PM). In this splicer, the splicing region is continuously purged by argon gas to stop the filament burning. This prevents contamination of the splice by solid deposits and water condensation, and also prevents combustion of flammable gases such as hydrogen and acetylene. For high fill pressures, the splice could be consolidated using heat-curable glue.

\section{Threshold measurements}

The energy threshold measurement for the generation of the first Stokes line $\mathrm{S}_{00}(1)$ was carried out in the following manner. The input power was varied by manual rotation of a half-wave plate, and the output light was collected and fed to an optical spectrum analyser and power detectors. The detected signal at the Stokes wavelength was monitored as the input power was increased up to a point where the Stokes signal emerged abruptly from the noise on the optical spectrum analyser. With this technique, we found a relative uncertainty varying between $40 \%$ and $70 \%$.

Received 3 November 2004; accepted 6 January 2005; doi:10.1038/nature03349.

1. Luiten, A. N. (ed.) Frequency Measurement and Control (Springer, Berlin, 2001).

2. Hall, J. L. Frequency stabilized lasers_a parochial review. Proc. SPIE 1837, 2-15 (1992).

3. Hentschel, M. et al. Attosecond metrology. Nature 414, 509-513 (2001).

4. Steinmeyer, G., Sutter, D. H., Gallmann, L., Matuschek, N. \& Keller, U. Frontiers in ultrashort pulse generation: Pushing the limits in linear and nonlinear optics. Science 286, 1507-1512 (1999).

Benabid, F., Knight, J. C., Antonopoulos, G. \& Russell, P. S. J. Stimulated Raman scattering in hydrogen-filled hollow-core photonic crystal fiber. Science 298, 399-402 (2002).

6. Benabid, F., Bouwmans, G., Knight, J. C., Russell, P. S. J. \& Couny, F. Ultra-high efficiency laser wavelength conversion in gas-filled hollow core photonic crystal fiber by pure stimulated rotational Raman scattering in molecular hydrogen. Phys. Rev. Lett. 93, 123903 (2004).

Russell, P. Photonic crystal fibers. Science 299, 358-362 (2003).

8. Marcuse, D. Loss analysis of single-mode fiber splices. Bell Syst. Tech. J. 55, 703-718 (1977).

9. Dushman, S. Scientific Foundations of Vacuum Technique (John Wiley \& Sons, New York, 1962).

10. Hansch, T. W. in The Hydrogen Atom (ed. Hansch, T. W.) 93-102 (Springer, Berlin, 1989).

11. Huber, A. et al. Hydrogen-deuterium 1S-2S isotope shift and the structure of the deuteron. Phys. Rev. Lett. 80, 468-471 (1998)

12. Quinn, T. J. Practical realization of the definition of the metre, including recommended radiations of other optical frequency standards (2001). Metrologia 40, 103-133 (2003).

13. Nakagawa, K., de Labachelerie, M., Awaji, Y. \& Kourogi, M. Accurate optical frequency atlas of the $1.5 \mu \mathrm{m}$ bands of acetylene. J. Opt. Soc. Am. B 13, 2708-2714 (1996).

14. Swann, W. C. \& Gilbert, S. L. Pressure-induced shift and broadening of 1510-1540-nm acetylene wavelength calibration lines. J. Opt. Soc. Am. B 17, 1263-1270 (2000).
15. MacAdam, K. B., Steinbach, A. \& Wieman, C. A narrow band tunable diode laser system with grating feedback, and a saturated absorption spectrometer for Cs and Rb. Am. J. Phys. 69, 1098-1111 (1992).

16. Wieman, C. E. \& Hollberg, L. Using diode lasers for atomic physics. Rev. Sci. Instrum. 62, 1-20 (1991).

17. Onae, A. et al. Optical frequency link between an acetylene stabilized laser at $1542 \mathrm{~nm}$ and an $\mathrm{Rb}$ stabilized laser at $778 \mathrm{~nm}$ using a two-color mode-locked fiber laser. Opt. Commun. 183, 181-187 (2000).

Acknowledgements The support of the UK Engineering and Physical Sciences Research Council (EPSRC) is acknowledged. F.B. thanks A. Luiten and M. Maric for discussions on frequency stabilization. F.B. is an EPSRC Advanced Research Fellow.

Competing interests statement The authors declare that they have no competing financial interests.

Correspondence and requests for materials should be addressed to F.B. (pysab@bath.ac.uk).

\section{Obliquity pacing of the late Pleistocene glacial terminations}

\section{Peter Huybers ${ }^{1} \&$ Carl Wunsch ${ }^{2}$}

${ }^{1}$ Woods Hole Oceanographic Institution, Woods Hole, Massachusetts 02543, USA

${ }^{2}$ Massachusetts Institute of Technology, Cambridge, Massachusetts 02139, USA

The 100,000-year timescale in the glacial/interglacial cycles of the late Pleistocene epoch (the past $\sim 700,000$ years) is commonly attributed to control by variations in the Earth's orbit ${ }^{1}$. This hypothesis has inspired models that depend on the Earth's obliquity ( $\sim 40,000 \mathrm{yr} ; \sim 40 \mathrm{kyr})$, orbital eccentricity ( $\sim 100 \mathrm{kyr})$ and precessional $(\sim 20 \mathrm{kyr})$ fluctuations ${ }^{2-5}$, with the emphasis usually on eccentricity and precessional forcing. According to a contrasting hypothesis, the glacial cycles arise primarily because of random internal climate variability ${ }^{6-8}$. Taking these two perspectives together, there are currently more than thirty different models of the seven late-Pleistocene glacial cycles?. Here we present a statistical test of the orbital forcing hypothesis, focusing on the rapid deglaciation events known as terminations ${ }^{10,11}$. According to our analysis, the null hypothesis that glacial terminations are independent of obliquity can be rejected at the $5 \%$ significance level, whereas the corresponding null hypotheses for eccentricity and precession cannot be rejected. The simplest inference consistent with the test results is that the ice sheets terminated every second or third obliquity cycle at times of high obliquity, similar to the original proposal by Milankovitch ${ }^{12}$. We also present simple stochastic and deterministic models that describe the timing of the latePleistocene glacial terminations purely in terms of obliquity forcing.

To test whether the glacial variability is related to changes in Earth's astronomical configuration, we adopt a formal nullhypothesis $\left(\mathrm{H}_{0}\right)$ that glacial terminations are independent of obliquity variations, and the alternative hypothesis $\left(\mathrm{H}_{1}\right)$ that glacial terminations are paced by it. Our focus on obliquity is motivated by previous indications of nonlinear interactions between obliquity period and quasi-100-kyr glacial variability ${ }^{13}$, but we also perform identical tests for pacing by precession and eccentricity. The test is focused on glacial terminations because their magnitude and abruptness facilitate accurate identification.

Several obstacles must be overcome to distinguish between $\mathrm{H}_{0}$ and $\mathrm{H}_{1}$. A major problem is the need to establish time controls on the glacial variability. Many studies estimate age by assuming a relationship between climate proxy variability and orbital 
forcing ${ }^{14,15}$, but this approach assumes the validity of the hypothesis being tested. Instead, we use an age-model devoid of orbital assumptions and apply it to the leading empirical orthogonal function (EOF1) of ten well-resolved marine $\delta^{18} \mathrm{O}$ records ${ }^{13}$, a proxy for ice volume and ocean temperature (Fig. 1a).

Most simple models of the late Pleistocene glacial cycles have at least four degrees of freedom ${ }^{2}$, and some have as many as twelve ${ }^{3}$. Unsurprisingly then, the seven observed quasi-100-kyr glacial cycles are insufficient to distinguish between the skill of the various models ${ }^{16}$. Models with minimal degrees of freedom are necessary. Other requirements for a useful test include the ability to cope with noisy records, age-model uncertainty and (possibly) nonlinear interactions. Here we test for stability in the phase of the orbital parameters during glacial terminations using Rayleigh's $R$ (see Methods).

To proceed, we must estimate the probability distribution function associated with $\mathrm{H}_{0}$. Of the five estimation methods explored, the one adopted gives the highest critical value and thus makes $\mathrm{H}_{0}$ the most difficult to reject-a modified random walk ${ }^{8}$ representing ice-volume variability:

$$
V_{t+1}=V_{t}+\eta_{t} \text { and if } V_{t} \geq T_{\mathrm{o}}, \text { terminate. }
$$

This highly simplified model posits 1-kyr steps in ice volume, $V_{t}$, of random length, $\eta_{t}$, independently drawn from a normal distribution with standard deviation $\sigma=2$ and mean $\mu=1$. The nonzero mean biases the Earth toward glaciation. Once $V_{t}$ reaches a threshold, $T_{\mathrm{o}}=90$, a termination is triggered, and ice-volume is linearly reset to zero over $10 \mathrm{kyr}$. If the model were deterministic with $\sigma=0$ and $\mu=1$, glacial cycles would last exactly $100 \mathrm{kyr}$, but with $\sigma=2$, glacial cycle duration is approximately normally distributed at $(100 \pm 20) \mathrm{kyr}$, a spread consistent with observations ${ }^{11}$. Initial ice volume is randomly set between 0 and $T_{\mathrm{o}}$ with equal probability. From a Monte Carlo technique (see Methods), we find a critical value of $R=0.60$.

The observed obliquity phases produce $R=0.70$, and $\mathrm{H}_{0}$ is rejected (Fig. 1b). This rejection of $\mathrm{H}_{0}$ is robust to all plausible

a
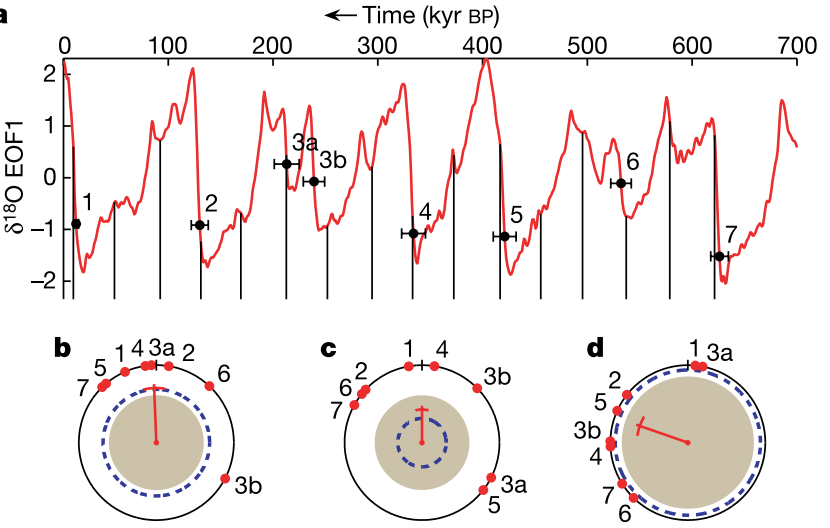

Figure 1 The Rayleigh test for phase directionality. a, $\delta^{18} 0$ EOF1 normalized so that negative values indicate more ice. Dots indicate onset of a termination and horizontal bars indicate one-standard-deviation age-model uncertainties ${ }^{13}$. Termination 3 is split between events $3 a$ and $3 b$. Vertical lines indicate the time of maxima in obliquity. Note that time runs from right to left in the palaeoclimate convention. $\mathbf{b}$, The obliquity phase (dots) sampled at each termination and plotted on a unit circle. The vector average has a magnitude $R=0.70$ (cross mark) exceeding the critical value $c=0.60$ (filled circle), so that $\mathrm{H}_{0}$ is rejected. Furthermore, $R$ is near $\mathrm{H}_{1}$ 's maximum-likelihood value (dashed circle). The direction is indistinguishable from maximum obliquity (top of the circle).

c, d, Analogous tests are made for precession $(R=0.43, C=0.60)$ (c) and eccentricity $(R=0.66, c=0.84)(\mathbf{d})$, but in neither case can the corresponding $\mathrm{H}_{0}$ be rejected. See the Supplementary Information for more details. reformulations of the test. Thus, the phase of obliquity has a statistically significant relationship with the timing of deglaciation. The mean phase at deglaciation is indistinguishable from zero and is associated with maxima in obliquity. We estimate the $H_{1}$ probability density function by assuming that terminations always initiate at the same phase of obliquity, but that termination timing is subject to identification and age-model uncertainties (see Methods). The maximum-likelihood value of $\mathrm{H}_{1}$ is $R=0.69$, very near the observed value, further supporting the conclusion that glacial terminations are paced by variations in obliquity. Hypotheses not accounting for the obliquity pacing are unlikely to be correct.

Analogous hypothesis tests for precession and eccentricity produce different results (Fig. 1c, d). Age-model uncertainty approaches half a precession cycle, so that the power of the precession test is negligible-even if present, precession pacing of the glacial cycles cannot be discerned. In the case of eccentricity, $\mathrm{H}_{0}$ is not rejected using the random-walk probability estimate (equation (1)), but is rejected using weaker formulations of the eccentricity null hypothesis. The discrepancy arises because null
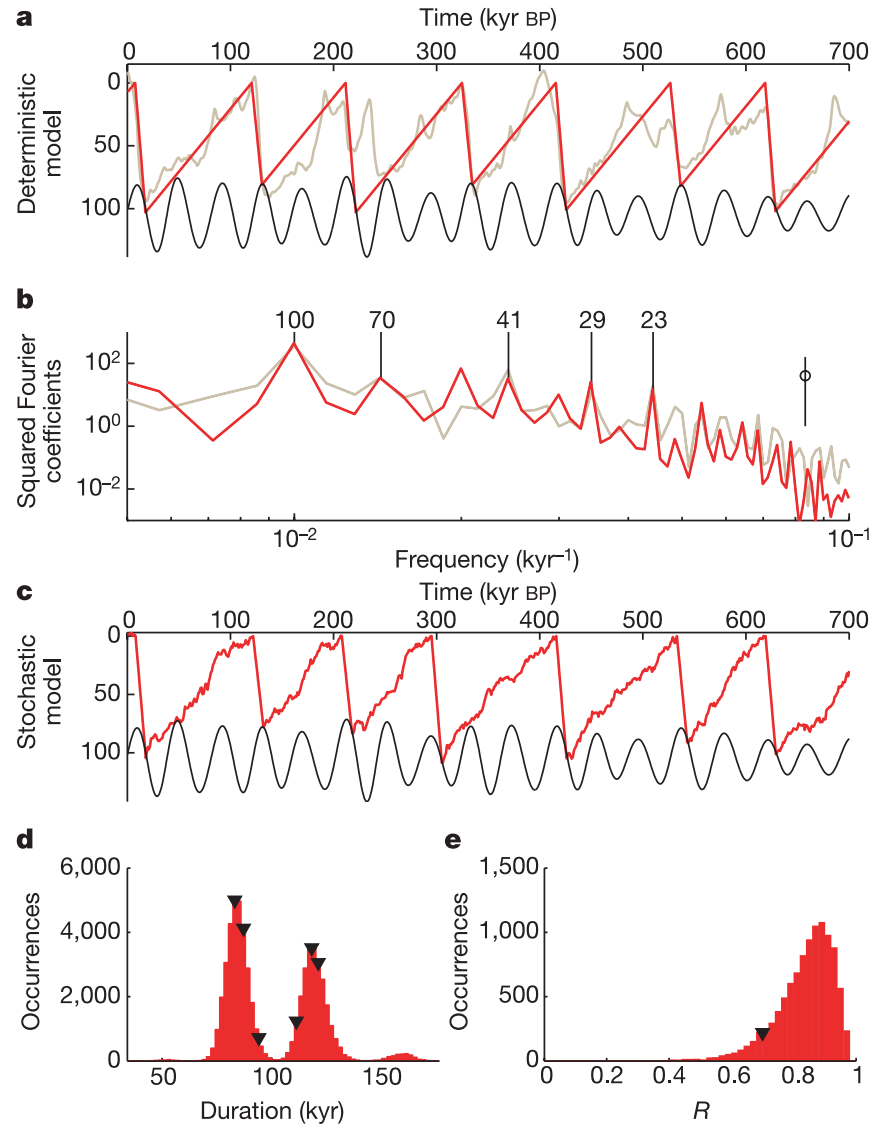

Figure 2 Deterministic and stochastic descriptions of the late-Pleistocene glacial variability. a, Deterministic model results (red) with an obliquity-dependent threshold (black) plotted over EOF1 (brown). b. Periodograms of the deterministic model results (red) and EOF1 (brown). Concentrations of energy are centred on the 1/41-kyr obliquity frequency and the 1/100-kyr glacial band; as well as combination tones at 1/70, 1/29 and $1 / 23 \mathrm{kyr}$. The approximate $95 \%$ confidence interval is indicated by the vertical bar on the right. c, A realization of the stochastic model. d, Histogram of the time between terminations, derived from many runs of the stochastic model. The observed duration between terminations (triangles, using termination 3a not 3b) coincide with the dominant 80- and 120-kyr modes. e, Histogram of Rayleigh's Rfrom the stochastic model with the observed obliquity value, $R=0.70$, indicated by the triangle. 
hypotheses that assume a glacial timescale of roughly $100 \mathrm{kyr}$ (which seem more physical) tend to have higher $R$ values and are more difficult to reject. Because the hypotheses of negligible influence of precession and eccentricity on the glacial terminations cannot be rejected, we adopt a minimalist strategy, retaining only obliquity to describe the glacial terminations.

From a physical standpoint, support for the obliquity control hypothesis also comes from the fact that maxima in obliquity cause annual average insolation anomalies of up to $10 \mathrm{Wm}^{-2}$ at high latitudes. Furthermore, the annual average and seasonal insolation redistributions associated with obliquity are hemispherically symmetric_as is the glacial variability to within a few thousand years ${ }^{17,18}$. Finally, although obliquity control does not by itself address the question of why termination five is anomalously large, it does alleviate the marine isotope stage 11 problem $^{2}$ of explaining the initiation of termination five when eccentricity and precession amplitude are small.

But how does a forcing with a 40-kyr period pace the 100-kyr latePleistocene glacial variability? One suggestion is that the phase and amplitude modulations of obliquity cause the 100-kyr variability ${ }^{4}$, but it is difficult to see the climatic significance of such modulations. Instead, we suggest that the climate state skips one or two obliquity beats before deglaciating, thus giving quantized glacial-cycle durations of either 80 or 120 kyr. A speculative scenario is for increased obliquity to increase high-latitude insolation and cause heating of an ice sheet, eventually warming the ice-bedrock interface. When the ice sheet is thin, basal temperature and pressure are low $^{19}$, and the obliquity heating has little effect-a skipped beat. But when the ice sheet is thick, basal temperature and pressure are high $^{19}$, and additional obliquity heating promotes basal melting. Enhanced lubrication of the ice-bedrock interface may trigger deglaciation by increasing ice flux into the ocean or toward lower latitudes, as well as by increasing the thinning rate and causing inward migration of the ablation zone $^{20}$. Note that $\sim 10 \mathrm{kyr}$ are required for surface heating to penetrate to the base of an ice sheet ${ }^{19}$. Unlike precession, changes in obliquity are associated with sustained annual average changes in insolation ${ }^{21,22}$ and are thus more likely to cause basal warming. Furthermore, if climate was warmer during the early Pleistocene, terminations would be triggered more nearly every obliquity cycle, giving the observed smaller and more rapid glacial variability $^{23}$.

A simple system, consistent with the above observations, is obtained by making the threshold in equation (2) dependent on obliquity:

$$
V_{t+1}=V_{t}+\eta_{t} \text { and if } V_{t} \geq T_{\mathrm{o}}-a \overline{\theta_{t}}, \text { terminate. }
$$

Here $\bar{\theta}_{t}$ is obliquity ${ }^{24}$, normalized to zero mean and unit variance, with amplitude $a$. The time-variable threshold makes it more likely for a termination to occur when obliquity is large. Both deterministic and stochastic variants of equation (2) are offered, to emphasize that such models are not theories of climate change, but rather attempts at efficient kinematic descriptions of the data, and that different mechanisms can be consistent with the limited observational records.

To make the model deterministic, the ice-volume step size in equation (2) is fixed at $\eta=1$. Setting $a=15, T_{\mathrm{o}}=105$ and (initial ice volume) $V_{(t=-700)}=30$ provides a good description of the late-Pleistocene glacial variability (Fig. 2a). Many other parameterizations yield similar results-we choose this one because it is particularly simple. The model produces the correct timing of the glacial terminations (using termination $3 a$, not $3 \mathrm{~b}$ ) and has a squared-cross-correlation with $\delta^{18} \mathrm{O}$ EOF1 of 0.65 , an excellent fit considering there are only three adjustable parameters. (Parameters other than $\eta$ could be adjusted instead.) For comparison, tuning other models having four ${ }^{2}$ or twelve $e^{3}$ adjustable parameters yields a maximum squared-cross-correlation with EOF1 of 0.24 and 0.74 respectively ${ }^{25}$. If skill is measured by squared-cross-correlation divided by the number of adjustable parameters, equation (2) does more than three times as well.

A periodogram of the deterministic model results (Fig. 2b) shows narrow-band concentrations of energy at the average 100-kyr period, the 41-kyr obliquity period, and at previously identified ${ }^{13}$ combination tones $1 / 41-1 / 100=1 / 70,1 / 41+1 / 100=1 / 29$, and $1 / 41+2 / 100=1 / 23 \mathrm{kyr}$-in good agreement with the $\delta^{18} \mathrm{O}$ EOF1 periodogram. The appearance of $1 / 23$-kyr narrow-band energy, in the absence of precession-band forcing, highlights the ambiguous origins of this energy band ${ }^{22}$. The model also has energy at $2 / 100 \mathrm{kyr}$, not visible in the observations. Note that the model produces an energetic background continuum consistent with the $\delta{ }^{18} \mathrm{O}$ periodogram even though it is deterministic.

For the stochastic case, $\eta_{t}$ is defined to be normally distributed with $\sigma=2$ and $\mu=1$. Here $\eta_{t}$ represents the unpredictable background weather and climate variability spanning all timescales out to the glacial/interglacial. All other parameter settings are kept the same as in the deterministic case. Now, equation (2) resembles an orderone autoregressive process. Such a process is known to describe the glacial variability well, except for runs associated with glacial terminations ${ }^{26}$, modelled here using the threshold condition in equation (2). The time between terminations in the stochastic model averages $100 \mathrm{kyr}$ but has a tri-modal distribution with maxima at two ( $80 \mathrm{kyr})$, three $(120 \mathrm{kyr})$, and four (160 kyr) obliquity cycles (Fig. 2d). The observed durations between terminations are consistent with the dominant 80- and 120-kyr modes, but suggest that one would see 160-kyr glacial cycles, given a larger sample size. The $R$ value obtained by the stochastic model averages 0.85 (Fig. 2e), higher than the observed $R=0.70$; this is as expected because of observational age-model error.

Deterministic and stochastic variants of equation (2) thus both describe the late-Pleistocene glacial variability. A description of the early Pleistocene variability ${ }^{23}$ is obtained by lowering the termination threshold to $T_{\mathrm{o}}=40$, giving smaller-amplitude terminations that occur more nearly every obliquity cycle. Alternatively, instead of specifying a parameter change, the mid-Pleistocene transition can be described using a chaotic model ${ }^{25}$ (not shown) having spontaneous transitions between 40 and 100-kyr modes of glacial variability. With only seven cycles, it is unclear whether adequate data will ever be available to distinguish between stochastic, simple deterministic and chaotic deterministic models of the glacial variability.

The simplest interpretation of our results is that during the Pleistocene epoch, the Earth tends to a glacial state (anthropogenic influences aside) and deglaciates near some, but not all, obliquity maxima. Obliquity control of the timing of deglaciation, probably in concert with a stochastic forcing, has several other consequences. These include inferences drawn from precession-based and eccentricity-based models of glaciation ${ }^{27}$, the hemispheric symmetry of glacial cycles, and the efficacy of age-model tuning of cores. These issues will be taken up elsewhere.

\section{Methods}

Rayleigh's $R$ is defined as ${ }^{28}$

$$
R=\frac{1}{N}\left|\sum_{n=1}^{N} \cos \phi_{n}+i \sin \phi_{n}\right|
$$

Here, $\phi_{n}$ is the phase of obliquity stroboscopically sampled at the $n$th glacial termination. The line brackets indicate the magnitude, making $R$ real and non-negative, with a maximum value of one when the phases are all equal. Relative to measures of phase coupling used to investigate other nonlinear interactions ${ }^{29}$, Rayleigh's $R$ requires many fewer phases (roughly five) to test for phase stability ${ }^{28}$.

To measure $R$ between obliquity and the glacial cycles, we use $\delta^{18} \mathrm{O}$ EOF1 and an agemodel independent of orbital assumptions ${ }^{13}$ (Fig. 1a). Such an independent age-model is important because even so-called minimal tuning strategies-using only a narrow band of a climate record-tend to align the abrupt glacial terminations with a particular phase of the assumed forcing (as indicated by Monte Carlo simulations), thus biasing records towards $\mathrm{H}_{1}$. 
The rate of change of EOF1 has a unimodal distribution with a long tail, indicative of rapid melting. Terminations are defined to initiate when the rate of change in EOF1 first exceeds the two-standard-deviation level. This criterion identifies each of the usual terminations $\mathrm{s}^{10,11}$, and two events in the termination 3 deglacial sequence (termed $3 \mathrm{a}$ and $3 \mathrm{~b}$ for the younger and older events, respectively). Additional rules could be added to exclude $3 \mathrm{a}$ or $3 \mathrm{~b}$, but this rejection seems ad hoc, and so we use all eight termination events. Note, the timing of termination 3 predicted by the Paillard model $^{3}$ also coincides with either event $3 \mathrm{a}$ or $3 \mathrm{~b}$, depending on slight changes in the parameterizations. Reassuringly, results are not sensitive to details of the test: either termination $3 \mathrm{a}$ or $3 \mathrm{~b}$ can be excluded; termination times can be defined using the midpoint between the local minimum and maximum bracketing each termination; and individual benthic or planktic $\delta^{18} \mathrm{O}$ records ${ }^{15}$ can be used in place of EOF1.

The probability density function (PDF) associated with $\mathrm{H}_{0}$ is estimated using the modified random-walk model (equation (1)). A realization of $R$ is obtained by sampling the phase of obliquity at eight consecutive termination initiations, generated from equation (1), and the PDF of $\mathrm{H}_{0}$ is estimated by binning $10^{4}$ such realizations of $R$. Other methods are to assume a uniform phase distribution, use surrogate data techniques ${ }^{30}$, or to derive statistics from ensemble runs of other models, but all of these give PDF estimates which make $\mathrm{H}_{0}$ more easily rejected and are therefore not used.

To estimate the PDF associated with $\mathrm{H}_{1}$, we assume that glacial terminations always occur at the same phase of obliquity, but that the phase observations are subject to identification and age-model error. A Monte Carlo technique is used where the timing of the glacial terminations are perturbed according to the estimated age uncertainties ${ }^{13}$ (these average $\pm 9 \mathrm{kyr}$ ) and identification error $( \pm 1 \mathrm{kyr}$, the EOF1 sampling resolution). A realization of $R$ is then computed using the phase of obliquity at the perturbed ages, and $10^{4}$ such realizations are binned to estimate the PDF of $\mathrm{H}_{1}$. We estimate the likelihood of correctly rejecting $\mathrm{H}_{0}$ (that is, the power of the obliquity test) to be 0.57 . See the Supplementary Information for a listing of the other pertinent data and statistics.

Received 6 August 2004; accepted 24 January 2005; doi:10.1038/nature03401.

1. Hays, J., Imbrie, J. \& Shackleton, N. Variations in the earth's orbit: Pacemaker of the ice ages. Science 194, 1121-1132 (1976)

2. Imbrie, J. \& Imbrie, J. Modeling the climatic response to orbital variations. Science 207, 943-953 (1980).

3. Paillard, D. The timing of Pleistocene glaciations from a simple multiple-state climate model. Nature 391, 378-391 (1998)

4. Liu, $\mathrm{H}$. Insolation changes caused by combination of amplitude and frequency modulation of the obliquity. J. Geophys. Res. 104, 25197-25206 (1999).

5. Gildor, H. \& Tziperman, E. Sea ice as the glacial cycles' climate switch: Role of seasonal and orbital forcing. Paleoceanography 15, 605-615 (2000).

6. Saltzman, B. Stochastically-driven climatic fluctuations in the sea-ice, ocean temperature, $\mathrm{CO}_{2}$, feedback system. Tellus 34, 97-112 (1982).

7. Pelletier, J. Coherence resonance and ice ages. J. Geophys. Res. 108, doi:10.1029/2002JD003120 (2003).

8. Wunsch, C. The spectral description of climate change including the 100ky energy. Clim. Dyn. 20, 353-363 (2003).

9. Saltzman, B. Dynamical Paleoclimatology: Generalised Theory of Global Climate Change (Academic, San Diego, 2002).

10. Broecker, W. Terminations. in Milankovitch and Climate (eds Berger, A. et al.) Part 2, 687-698 (D. Riedel, Hingham, 1984).

11. Raymo, M. E. The timing of major climate terminations. Paleoceanography 12, 577-585 (1997).

12. Milankovitch, M. Kanon der Erdbestrahlung und seine Andwendung auf das Eiszeiten-problem (Royal Serbian Academy, Belgrade, 1941).

13. Huybers, P. \& Wunsch, C. A depth-derived Pleistocene age-model: Uncertainty estimates, sedimentation variability, and nonlinear climate change. Paleoceanography 19, doi:10.1029/ 2002PA000857 (2004).

14. Imbrie, J. et al. in Milankovitch and Climate (eds Berger, A. et al.) Part 1, 269-305 (D. Riedel Publishing Company, 1984)

15. Shackleton, N. J., Berger, A. \& Peltier, W. R. An alternative astronomical calibration of the lower Pleistocene timescale based on ODP site 677. Trans. R. Soc. Edinb. Earth Sci. 81, 251-261 (1990).

16. Roe, G. \& Allen, M. A comparison of competing explanations for the 100,000-yr ice age cycle. Geophys. Res. Lett. 26, 2259-2262 (1999).

17. Blunier, T. \& Brook, E. Timing of millennial-scale climate change in Antarctica and Greenland during the last glacial period. Science 291, 109-112 (2001).

18. Wunsch, C. Greenland-Antarctic phase relations and millennial time-scale climate fluctuations in the Greenland cores. Quat. Sci. Rev. 22, 1631-1646 (2003).

19. Marshall, S. \& Clark, P. Basal temperature evolution of North American ice sheets and implications for the 100-kyr cycle. Geophys. Res. Lett. 29, doi:10.1029/2002GL015192 (2002).

20. Zwally, H. et al. Surface melt-induced acceleration of greenland ice-sheet flow. Science 297, 218-222 (2002).

21. Rubincam, D. Insolation in terms of earth's orbital parameters. Theor. Appl. Climatol. 48, 195-202 (1994).

22. Huybers, P. \& Wunsch, C. Rectification and precession-period signals in the climate system. Geophys. Res. Lett. 30, doi:10.1029/2003GL017875 (2003).

23. Raymo, M. \& Nisancioglu, K. The 41 kyr world: Milankovitch's other unsolved mystery. Paleoceanography 18, doi:10.1029/2002PA000791 (2003).

24. Berger, A. \& Loutre, M. F. Astronomical solutions for paleoclimate studies over the last 3 million years. Earth Planet. Sci. Lett. 111, 369-382 (1992).

25. Huybers, P. On the Origins of the Ice Ages: Insolation Forcing, Age Models, and Nonlinear Climate Change. PhD thesis, MIT (2004).

26. Wunsch, C. Quantitative estimate of the Milankovitch-forced contribution to observed quaternary climate change. Quat. Sci. Rev. 23, 1001-1012 (2004).
27. Ruddiman, W. F. Oribital insolation, ice volume, and greenhouse gases. Quat. Sci. Rev. 22, 1597-1622 (2003)

28. Upton, G. \& Fingleton, B. Spatial Data Analysis by Example Vol. 2 (John Wiley and Sons, Chichester, 1989).

29. Rosenblum, M. \& Pikovsky, A. Synchronization: from pendulum clocks to chaotic lasers and chemical oscillators. Contemp. Phys. 44, 401-416 (2003).

30. Schreiber, T. \& Schmitz, A. Surrogate time series. Physica D 142, 346-382 (2000).

Supplementary Information accompanies the paper on www.nature.com/nature

Acknowledgements Useful comments were provided by E. Boyle, W. Curry, T. Herbert, J. McManus, F. Ng, M. Tingley and G. Yang. P.H. is supported by the NOAA Postdoctoral Program in Climate and Global Change and C.W. is supported in part by the National Ocean Partnership Program (ECCO).

Competing interests statement The authors declare that they have no competing financial interests.

Correspondence and requests for materials should be addressed to P.H. (phuybers@whoi.edu).

\section{Two episodes of microbial change coupled with Permo/Triassic faunal mass extinction}

\author{
Shucheng Xie ${ }^{1,2}$, Richard D. Pancost ${ }^{1}$, Hongfu Yin ${ }^{2}$, Hongmei Wang ${ }^{3}$ \\ \& Richard P. Evershed
}

${ }^{1}$ Organic Geochemistry Unit, Bristol Biogeochemistry Center, School of Chemistry, University of Bristol, Cantock's Close, Bristol BS8 1TS, UK

${ }^{2}$ Faculty of Earth Science and State Key Laboratory of Geological Processes and Mineral Resources, China University of Geosciences, Wuhan 430074, People's

Republic of China

${ }^{3}$ School of Environmental Studies, China University of Geosciences, Wuhan 430074, People's Republic of China

Microbial expansion following faunal mass extinctions in Earth history can be studied by petrographic examination of microbialites (microbial crusts) ${ }^{1,2}$ or well-preserved organic-walled microbes ${ }^{3}$. However, where preservation is poor, quantification of microbial communities can be problematic. We have circumvented this problem by adopting a lipid biomarker-based approach to evaluate microbial community changes across the Permo/Triassic $(\mathrm{P} / \mathrm{Tr})$ boundary at Meishan in South China. We present here a biomarker stratigraphic record showing episodic microbial changes coupled with a high-resolution record of invertebrate mass extinction. Variation in the microbial community structure is characterized by the 2-methylhopane (2-MHP) index (a ratio of the abundance of cyanobacterial biomarkers to more general bacterial biomarkers). Two episodes of faunal mass extinction were each preceded by minima in the 2-MHP index, followed by strong maxima, likely reflecting microbial responses to the catastrophic events that caused the extinction and initiated ecosystem changes. Hence, both cyanobacterial biomarker and invertebrate fossil records provide evidence for two episodes of biotic crisis across the $\mathrm{P} / \mathrm{Tr}$ boundary.

In bacteria, bacteriohopanepolyols (BHPs) are amphiphilic biochemicals that serve regulatory and rigidifying functions in cell membranes. It has been demonstrated that the cyanobacterial lineage currently comprises the only known quantitatively significant source of 2-methyl-BHPs ${ }^{4-6}$, such that the presence of abundant $2 \alpha$-methylhopanes (2-MHPs) derived from 2-methyl-BHP precursors is believed to be characteristic of cyanobacteria ${ }^{4}$. This is particularly true for 2-methyl-BHPs containing more than 32 carbon atoms, which have not been detected in organisms other 